\title{
Niveles de parasitemia y alteraciones histopatológicas en Mus musculus BALB/c infectado con Trypanosoma cruzi obtenido de Panstrongylus chinai del Valle Chamán, La Libertad - Perú
}

\author{
ENRIQUE DÍAZ-LIMAY*, HERMES ESCALANTE* y CÉSAR A. JARA*
}

\begin{abstract}
PARASITEMIA LEVELS AND HISTOPATHOLOGYCAL CHANGES IN Mus musculus BALB/C INFECTED WITH Typanosoma cruzi OBTAINED FROM Panstrongylus chinai OF CHAMAN VALLEY, LALIBERTAD - PERU
\end{abstract}

Parasitemia levels as well as histopathologycal changes in the heart and the brain of mice, Mus musculus, BALB/c experimentally infected by Trypanosoma cruzi isolated of Panstrongyfus chinai from Chaman Valley (La Libertad-Peru) was determined. For this purposes, ten mice specimens was inoculated with $5 \times 10^{4}$ trypomastigota parasites $(0.1 \mathrm{~mL}$ of original inoculums $)$ and another one, the witness, was inoculated with saline solution sterile. The parasitemia was evident at fifth day

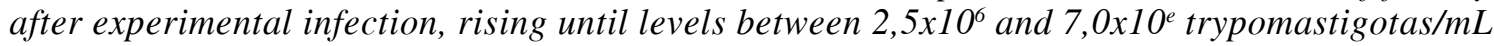
before death, that which happened in 100\% of experimental mice between 13 to 18 days. On the other hand, the histopathologycal analysis by H/E technique showed edematous areas, vesicular congestion, hipercromasia and amastigota nests in both, heart and encephalon cuts. Moreover, only in encephalon chronic inflammation was detected and only in hearth, lynphocyte-inflamatory infiltred. T. cruzi of $\boldsymbol{P}$. chinai bug from Chaman Valley showed neurotropic and cardio tropic tendency in M. musculus BALB/c.

Key words: Trypanosoma cruzi, Panstrongylus chinai, histopathological changes, parasitemia.

\section{INTRODUCCIÓN}

La enfermedad de Chagas o tripanosomiosis americana es una zoonosis propia del continente americano producida por Trypanosoma cruzi y transmitida por insectos hematófagos de la subfamilia Triatominae ${ }^{1,2}$ constituyendo un serio problema de salud pública porque aproximadamente 18 millones de personas están infectadas, de las cuales, entre 23 y $43 \mathrm{mil}$ personas mueren al año ${ }^{3}$. Esta parasitosis se desarrolla en tres fases definidas: la aguda, que se presenta con fiebre, dolores de cabeza, agrandamiento de ganglios, hígado y bazo; la latente, sin presentación clínica debido a que la reacción inmunológica del hospedero logra controlar la reproducción del parásito y la crónica, en la que se presentan alteraciones del sistema nervioso de diversos órganos, particularmente el corazón y tubo digestivo donde

\footnotetext{
* Departamento de Microbiología y Parasitología. Universidad Nacional de Trujillo. Trujillo-Perú.
} 
se originan los denominados megas ${ }^{4-6}$.

En el Perú, la zona costera sur (Ica, Arequipa, Moquegua, Tacna) es el área donde se han presentado la mayoría de casos de Enfermedad de Chagas, siendo Triatoma infestans el vector y Cavia porcellus "cobayo" el principal reservorio ${ }^{7}$. En la zona norte, en cambio, se han registrado pocos casos humanos ${ }^{8}$. No obstante, investigaciones efectuadas en las dos últimas décadas han permitido conocer que existen alrededor de 17 especies de triatominos, de las cuales Panstrongylus chinai es la más común ${ }^{9}$, habiendo sido encontrada naturalmente infectada con $T$. cruzi en algunas zonas ${ }^{10-13}$, al igual que Rattus norvegicus, $C$. porcellus y Canis familiaris ${ }^{14,15}$, y que pobladores y varias especies de animales de distintas zonas han mostrado serología positiva a este parásito ${ }^{16-18}$.

El T. cruzi aislado de $P$. chinai de algunas localidades costeras del norte peruano posee distinto comportamiento patogénico en ratones isogénicos, siendo los ejemplares jóvenes de la cepa BALB/c el modelo más atractivo por su alta sensibilidad y porque en él se puede reproducir la enfermedad de modo semejante a como se presenta en el hombre ${ }^{4}$. Utilizando este modelo animal se ha establecido que $T$. cruzi del valle de Reque produjeron elevada parasitemia, así como, edema, congestión, gliosis y necrosis multifocal en los órganos parasitados ${ }^{19}$ y que los procedentes de Callanca (Lambayeque) también produjeron elevada parasitemia, anemia, leucopenia y linfocitopenia ${ }^{20}$. Sin embargo, se desconoce si T. cruzi de otros valles, entre ellos el de Chamán, produce manifestaciones compatibles con las encontradas en Reque y Callanca u otras, en el mismo modelo animal. El conocimiento de este aspecto resulta importante, porque las diferencias en cuanto al comportamiento patogénico de las cepas distintas, podría correlacionarse con el curso de la enfermedad en humanos ${ }^{3,6}$.

El presente trabajo estuvo orientado a determinar los niveles de parasitemia y alteraciones histopatológicas producidas en el encéfalo y corazón de $M$. musculus cepa BALB/ $\mathrm{c}$ en el curso de una infección experimental con $T$. cruzi obtenido de P. chinai procedentes del Valle de Chamán, La Libertad-Perú.

\section{MATERIAL Y MÉTODOS}

Animales de experimentación: Se utilizó
10 ejemplares de $M$. musculus BALB/c isogénicos machos, de 20 días de edad, obtenidos en el bioterio del Departamento de Microbiología y Parasitología de la Universidad Nacional de Trujillo, a partir de padres procedentes del Instituto Nacional de Salud (Lima). Uno de ellos se empleó como control y los nueve restantes conformaron el grupo experimental.

Parásitos: De dos de los ratones usados para mantener la "cepa" de T. cruzi obtenida una semana antes de $P$. chinai capturado en ambientes peridomiciliarios en el valle Chaman (Chepen, La Libertad-Perú), cuando se encontraron con un nivel de parasitemia elevado, se extrajo sangre mediante punción cardíaca para ser diluida en solución salina fisiológica estéril (SSFE), de modo tal que $0,1 \mathrm{~mL}$ contenga una concentración aproximada de $5 \times 10^{4}$ tripomastigotas $^{21}$. Esta suspensión se inóculo intraperitonealmente a cada uno de los ratones del grupo experimental, mientras que al ratón control se incoculó, por la misma vía, $0,1 \mathrm{~mL}$ de SSFE.

Evaluación de parasitemias y de las alteraciones histopatológicas: Durante el transcurso de la experiencia se evaluó los signos clínicos evidenciables a simple vista y se determinaron los niveles de parasitemia al $5^{\circ}$, $10^{\circ}$ y $15^{\circ}$ día de la inoculación. Para esto, se obtuvo sangre del la parte distal de la cola utilizando EDTA como anticoagulante, a una dilución 1/4.000 en una pipeta de recuento de leucocitos determinándose la cantidad de parásitos por unidad de volumen en cámara deNeubauer ${ }^{21,22}$.

Los ratones sacrificados a partir del décimo tercer día de la infección y los que murieron por efecto de la enfermedad fueron necropsiados para extraer el encéfalo y el corazón, los cuales fueron fijados y conservados en formol al $10 \%$ con el fin de efectuar estudios anatomopatológicos, mediante la técnica $\mathrm{H} / \mathrm{E}$ convencional.

\section{RESULTADOS}

Se encontró tripomastigotas de $T$. cruzi en la sangre de los ratones del grupo experimental a partir del quinto día de la inoculación (Tabla 1), cuyos niveles de parasitemia variaron desde 0,20 hasta $0,25 \times 10^{3}$ tripomastigotas $/ \mathrm{mL}$ de sangre. Al décimo día de infección se evidenció que los niveles de parasitemia aumentaron hasta $3,5 \times 10^{\text {s }}$, 
mostrando sus máximos niveles al décimo quinto día post-inoculación con valores máximos de 7,0 x $10^{6}$. Se observó, así mismo, que todos los ratones infectados $(100 \%)$ murieron, hecho que ocurrió entre los 13 y 18 días de la inoculación (Tablas 1 y 2).

La totalidad de los ratones del grupo experimental presentaron diferente comportamiento en relación al ratón control. Se observó inapetencia progresiva, erizamiento del pelo más notorio en la parte de la nuca, incontinencia frecuente de la orina, parálisis de las extremidades posteriores $\mathrm{y}$, finalmente, convulsiones hasta producirse la muerte, permaneciendo vivo el ratón control.
El estudio macroscópico de los órganos de los animales muertos, comparado con el control, mostró aumento de tamaño del corazón y el encéfalo, así como, congestión, edema en dichos órganos.

El estudio histológico del encéfalo mostró, edema, congestión vascular, células gliales y pseudoquistes conformados por grupos de amastigotas de $T$. cruzi. (Figura 1). En el miocardio se observó pseudoquistes conformados por grupos de amastigotas que ocasionan fragmentación de las fibras musculares cardíacas, áreas de edema, congestión vascular, hipercromasia e infiltrado inflamatorio linfocitario (Figura 2).

Tabla 1. Niveles de parasitemia en Mus musculus BALB/c infectado con tripomastigotas de Trypanosoma cruzi obtenido de Panstrongylus chinai del valle de Chamán, La Libertad-Perú

\begin{tabular}{|c|c|c|c|c|c|}
\hline \multirow[b]{2}{*}{ Ratones } & \multicolumn{4}{|c|}{ Niveles de Parasitemia (Tripomastigotas/mL de sangre) } & \multirow[t]{2}{*}{$\mathbf{N}^{\circ}$ días de sobrevida } \\
\hline & $1^{\circ}$ día & $5^{\circ}$ día & $10^{\circ}$ día & $15^{\circ}$ día & \\
\hline E1 & 0,0 & $0,25 \times 10^{5}$ & $2,5 \times 10^{6}$ & $3,5 \times 10^{6}$ & 18 \\
\hline $\mathrm{E} 2$ & 0,0 & $0,25 \times 10^{5}$ & $2,5 \times 10^{6}$ & $3,0 \times 10^{6}$ & $18 *$ \\
\hline E3 & 0,0 & $0,25 \times 10^{5}$ & $3,5 \times 10^{6}$ & murió & 13 \\
\hline $\mathrm{E} 4$ & 0,0 & $0,25 \times 10^{5}$ & $2,5 \times 10^{6}$ & $5,5 \times 10^{6}$ & $17 *$ \\
\hline E5 & 0,0 & $0,25 \times 10^{5}$ & $2,5 \times 10^{6}$ & $7,0 \times 10^{6}$ & $16^{*}$ \\
\hline E6 & 0,0 & $0,25 \times 10^{5}$ & $3,5 \times 10^{6}$ & murió & 13 \\
\hline E7 & 0,0 & $0,25 \times 10^{5}$ & $4,5 \times 10^{6}$ & murió & 15 \\
\hline E8 & 0,0 & $0,25 \times 10^{5}$ & $2,5 \times 10^{6}$ & $4,5 \times 10^{6}$ & 15 \\
\hline E9 & 0,0 & $0,25 \times 10^{5}$ & $2,5 \times 10^{6}$ & murió & 14 \\
\hline $\mathrm{C}$ & 0,0 & 0,0 & 0,0 & vivió & $15 *$ \\
\hline
\end{tabular}

$\mathrm{E}=$ Ratón experimental; $\mathrm{C}=$ Ratón control; * = Número de días al cual se sacrificó

Tabla 2. Signos clínicos, niveles de parasitemia y tiempo de muerte en Mus musculus cepa BALB/c Infectados con Trypanosoma cruzi obtenido de Panstrongylus chinai del valle de Chamán - La Libertad

\begin{tabular}{|c|c|c|c|}
\hline Ratones & & $\underset{\text { Signos }}{\text { Parasitemia al } 15^{\circ} \text { día }}$ & $\begin{array}{l}\text { Tiempo de vida } \\
\left(\text { trip/mL de sangre } \times 1^{6}\right)(\text { días })\end{array}$ \\
\hline E1 & $a, b, c, d$ & 3,5 & 18 \\
\hline $\mathrm{E} 2$ & $a, b, c, d$ & 3,0 & 18 \\
\hline E3 & $a, b, c, d$ & M & 13 \\
\hline $\mathrm{E} 4$ & $a, b, c, d$ & 5,5 & 17 \\
\hline E5 & $a, b, c, d$ & 7,0 & 16 \\
\hline E6 & $a, b, c, d$ & $\mathrm{M}$ & 13 \\
\hline E7 & $a, b, c, d$ & $\mathrm{M}$ & 15 \\
\hline E8 & $a, b, c, d$ & 4,5 & 15 \\
\hline E9 & $a, b, c, d$ & $\mathrm{M}$ & 14 \\
\hline $\mathrm{C}$ & - & 0,0 & 15 \\
\hline
\end{tabular}

$\mathrm{E}=$ ratones experimentales; $\mathrm{C}=$ ratón control; $\mathrm{M}=$ muerte por enfermedad o sacrificio; $\mathrm{a}=$ erizamiento del pelo; $\mathrm{b}=$ incontinencia de orina; $\mathrm{c}=$ parálisis de extremidades posteriores; $\mathrm{d}=$ convulsiones marcadas; - = ausencia de signo. 


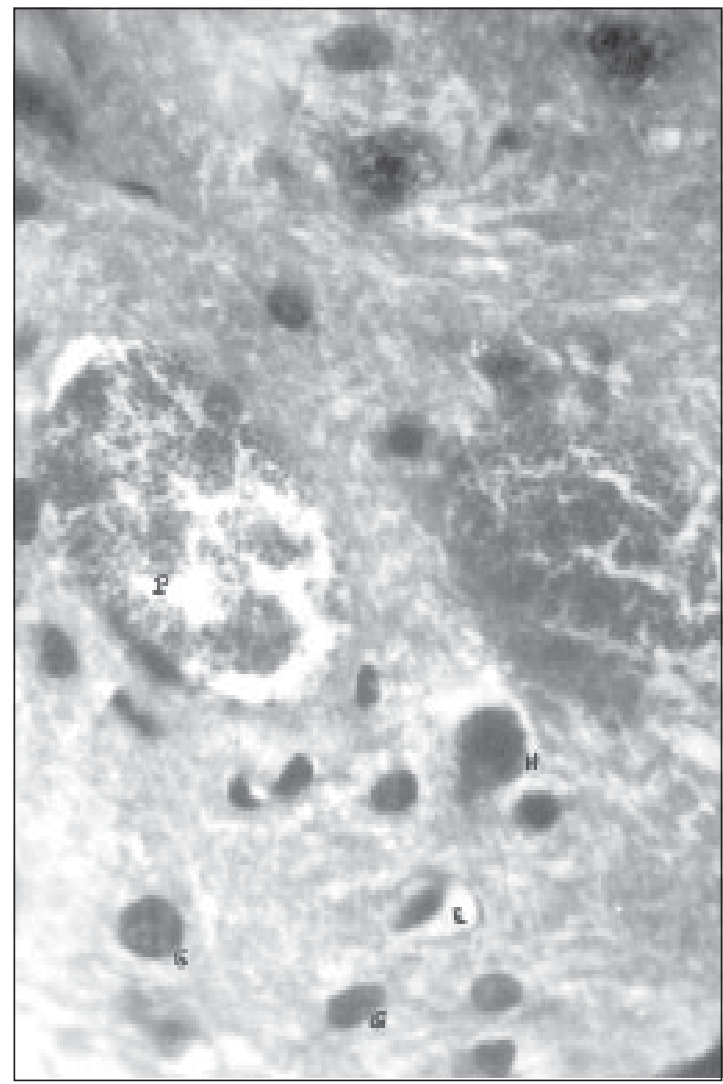

Figura 1. Corte histológico del encéfalo de Mus musculus BALB/c infectado experimentalmente con Trypanosoma cruzi obtenido de Panstrongylus chinai del valle Chamán, La Libertad-Perú (Col H/E). a = pseudoquistes (nido de amastigota); $\mathrm{b}=$ edema; $\mathrm{c}=$ células gliales; $\mathrm{d}=$ congestión vascular.

\section{DISCUSIÓN}

En el presente trabajo se utilizaron ratones $\mathrm{BALB} / \mathrm{c}$ machos debido a que estudios previos han permitido determinar que, junto con otras cepas como la $\mathrm{C}_{3} \mathrm{H} / \mathrm{HeN}$ y la $\mathrm{C}_{57} \mathrm{BL} / 6$, se hallan entre las más susceptibles a la infección por $T$. cruzi, particularmente los machos ya que en las hembras los estrógenos estimulan la actividad fagocítica de los macrófagos incrementando la respuesta inmune local ${ }^{5,23,25}$. Se confirma entonces la elevada susceptibilidad de esta cepa murina en base a la elevada parasitemia, el 100\% de mortalidad y daños histológicos encontrados.

La presentación clínica, los parámetros de patogenicidad, los niveles de parasitemia y los cambios histológicos son semejantes a lo observado en dos investigaciones efectuadas con

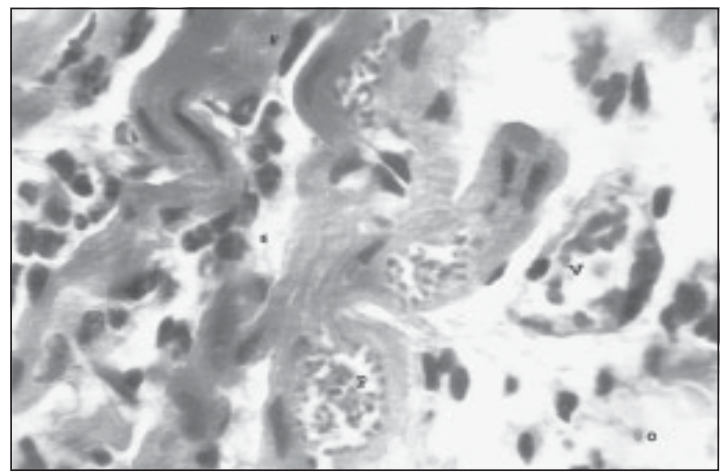

Figura 2. Corte histológico del corazón de Mus musculus BALB/c infectado experimentalmente con Trypanosoma cruzi obtenido de Panstrongylus chinai del valle Chamán La LibertadPerú. $\mathrm{a}=$ pseudoquistes (nido de amastigotas); $\mathrm{b}=$ congestión vascular; $\mathrm{c}=$ edema; $\mathrm{e}=$ infiltrado inflamatorio linfocitario.

T. cruzi también aislado de $P$. chinai, pero de Reque y de Callanca, lugares ubicados en el departamento de Lambayeque que limita con el de La Libertad ${ }^{21,22}$. Esta semejanza permitiría afirmar que se está frente a la misma cepa del protozoario, debido a que proceden del mismo insecto que habita en valles ubicados en la misma zona geográfica, muy cercanos unos de otros, con las mismas características ecológicas y por lo tanto, con la presencia de los mismos cultivares y las mismas especies de animales sinantrópicos.

La presencia de nidos de amastigotas y de los demás daños histopatológicos a nivel de encéfalo y corazón muestra una relación directa con el nivel de parasitemia alcanzado, lo que indica que los parásitos después de instalarse y reconocer los diferentes tejidos se reproducen intensamente a nivel intracelular, dando lugar a cada vez mayor número de parásitos en sangre luego de diferenciarse en tripomastigotas. Esta secuencia no se observa en todas las cepas de $T$. cruzi, procedentes de Chile, Argentina o Brasil en donde prevalece la tendencia macrofagotropica o reticulotropica en infecciones experimentales en el mismo modelo animal, sin causar daños a los tejidos ${ }^{5,23,24}$. Estas diferencias entre las distintas cepas de diversas zonas geográficas confirman la gran variedad de cepas de este parásito, no sólo desde el punto de vista morfofisiológico, sino también patogénico tratándose de un complejo denominado "cruzi".

Las convulsiones y la parálisis de los miembros posteriores observados ya han sido registrados 
anteriormente para diferentes cepas de $T$. cruzi. Se ha determinado que las convulsiones y la parálisis están relacionadas con el deterioro de algunas partes del sistema nervioso central ocasionado por el parásito ${ }^{21}$ y por el daño que el parásito causa en el cerebro y médula espinal, por ser éstos los órganos responsables del comando de la fisiología de la vejiga en dichos huéspedes ${ }^{25}$; además, es posible que ello se produzca debido a daño directo en dicho órgano, o en su defecto al entrar en estado de inestabilidad próximas a la muerte. Por su parte, el erizamiento de pelo tendría una relación indirecta con el SNC ya que las lesiones directas causadas por el parásito se realizan en la periferia de dicho órgano $^{21,25}$.

Los signos clínicos observados en los ratones estudiados conllevaría a pensar que se trata de una cepa neurotrófica, aunque algunos otros signos observados, como la muerte súbita del $100 \%$ de los ratones, estaría relacionado además por el daño producido por el parásito a nivel cardíaco, desencadenándose alguna variedad de arritmia hecho que también ha sido observado en Brasil ${ }^{23}$.

La presencia de nidos de amastigota de $T$. cruzi tanto en encéfalo como en corazón indican que el parásito está directamente involucrado al daño ocasionado. Entonces, las reacciones histológicas ocurren por la extravazación del plasma sanguíneo que da lugar a edemas, destrucción nuclear, seguidas por hipercromia y retracción nuclear (picnosis). Por su lado, la necrosis multifocal se debe a modificaciones morfológicas causadas directamente también por el parásito que conlleva inevitablemente a la muerte de las células nerviosos ${ }^{21-23}$.

Lo señalado conlleva a afirmar que es indiscutible la acción de T. cruzi sobre el encéfalo y el corazón y que todos los órganos pueden ser afectados durante la infección ocasionándose aumento del tamaño de diversos órganos, tales como, bazo e hígado, siendo, sin embargo, aquellas que dañan el SNC las cepas más virulentas 6 . De modo que los resultados obtenidos en la presente investigación permite ubicar al $T$. cruzi del valle de Chamán. La Libertad, dentro de estas últimas cepas, ya que la invasión por la forma de amastigota en este sistema fue claramente verificada.

\section{RESUMEN}

Se determinó los niveles de parasitemia y las alteraciones histopatológicas en el corazón y el encéfalo de Mus musculus cepa BALB/c infectado experimentalmente con Trypanosoma cruzi obtenido de Panstrongyius chinai capturados en el valle de Chamán, La Libertad-Perú.

El trabajo se hizo en 10 ejemplares de $M$. musculus machos (nueve experimentales y un control) de 20 días de edad. A cada uno de los ejemplares del grupo experimental se les inoculó intraperitonealmente $5 \times 10^{4}$ tripomastigotes $(0,1 \mathrm{~mL}$ inoculo original) suspendidos en sangre, $\mathrm{y}$ al control $0,1 \mathrm{~mL}$ de solución salina fisiológica estéril.

La parasitemia se inició a partir del quinto día de la inoculación del T. cruzi, elevándose hasta niveles comprendidos entre $2,5 \times 10^{6}$ y $7,0 \times 10^{6}$ tripomastigotes $/ \mathrm{mL}$, antes de la muerte de los animales (18 días como máximo). El corazón y encéfalo fueron separados y conservados en formol al 10\% para realizar cortes y colorearlos con la técnica $\mathrm{H} / \mathrm{E}$.

Todos los ratones parasitados presentaron: erizamiento de pelo, incontinencia urinaria, parálisis de extremidades posteriores y convulsiones. A nivel histopatológico, tanto en el corazón como en el encéfalo, se observaron áreas de edema, congestión vascular, hipercromasia y nidos de amastigota. En el encéfalo se presentó, además, focos de inflamación crónica y difusa y en el corazón infiltrado inflamatorio linfocitario.

\section{REFERENCIAS}

1.- NÁQUIRA C, HUAPAYA P, ESPINOSA Y, VEGA S. Enfermedad de Chagas. Ministerio de Salud, LimaPerú; 2001

2.- TANOWITZ H B, KIRCHHOFF L V, SIMON D et al. Chagas’ Disease. Clin Microbiol Rev 1992; 5: 400-9.

3.- APT W, REYES H. Algunos aspectos de la Enfermedad de Chagas en Latinoamérica. Parasitol al Día 1990; 14: 23-40.

4.- ANTAS PRZ, MEDRANO-MERCADO F, TORRICO F, et al. Early, intermediate and late acute stages in Chagas' Disease: a study combining anti-galactose IgG, specific serodiagnosis, and polymerase chain reaction analysis. Am J Trop Med Hyg 1999; 6: 308-14.

5.- ZÚÑIGA C, PARRA MA, VELA $H$ et al. Estudio histopatológico en ratones infectados experimentalmente con Trypanosoma cruzi. Parasitol al Día 1998; 22: 45-9.

6.- DEVERA R, FERNANDEZ O, COURA J R. Should Trypanosoma cruzi be called "cruzi Complex? A review of the parasite diversity and the potential of selecting population after in vitro culturing and mice infection. Mem Inst Oswaldo Cruz, Rio de Janeiro 2003; 98: 112.

7.- AYAQUI R, CÓRDOVA E. Epidemiología de la Enfermedad de Chagas en el Valle de Vítor (Distrito 
Vítor, Departamento de Arequipa). 1984. Bol Peruano Parasit 1982-1988; 4: 19-24.

8.- VASALLO C. Casos autóctonos de la Enfermedad de Chagas descubiertos en el Dpto de Piura. Acta Méd Per 1982; 9: 17-24.

9.- GUILLEN Z, CÁCERES I, ELLIOT A, RAMÍREZ J. Triatominos del norte-peruano y su importancia como vectores de Trypanosoma spp. Rev Per Ent 1988; 31: 21-30.

10.-PAREDES Y, TAPIA G. Determinación de triatominos y su infección natural por Trypanosoma cruzi en el caserío de Callanca Monsefú, Lambayeque. (Tesis Lic. en Biología). UN Pedro Ruiz-Gallo. Lambayeque. 1991.

11.- JARA C A, ESCALANTE H, ROLDAN J, DÍAZ E. Distribución y frecuencia de infección por Trypanosoma cruzi de triatominos y Ovis aries en el valle Chamán, La Libertad-Perú. SCIENDO 1998; 1: 23-33.

12.- ESCALANTE H, LÓPEZ-ROSAS E, JARA C A, TORRES-LUNA P. Distribución de la Enfermedad de Chagas en la zona norte del Perú. REBIOL 1997; 17: 115-29.

13.- ARMAS M. Distribución de triatominos y su infección por Trypanosoma cruzi en los distritos de Magdalena, Chilete y Yonán (Departamento de Cajamarca). (Tesis Bachiller). Facultad de Ciencias Biológicas de la Universidad Nacional de Trujillo. 1992.

14.- ARANA O, CASTILLO R. Reservorios naturales de Trypanosoma cruzi en los distrito de Magdalena y Chilete, Dpto. de Cajamarca. REBIOL 1994; 13: 49-53.

15.- JARA C A, ESCALANTE H, ROLDAN J et al. Frecuencia de infección por Trypanosoma cruzi en pobladores y animales sinantrópicos del valle Chamán, La Libertad-Perú. SCIENDO 1999; 2: 21-33.

16.- JARA C A, ESCALANTE H, DÍAZ-LIMAY E, J ROLDÁN. Aspectos epidemiológicos de la enfermedad de Chagas en el valle de Zaña, Lambayeque-Perú. SCIENDO 2002; 5: 9-21.

17.- GUTIÉRREZ I, MURILlo V. Detección de anticuerpos IgG anti-Trypanosoma cruzi por inmunofluorescencia indirecta en pobladores del valle Reque-Lambayeque. (Tesis Bachiller). Facultad de Ciencias Médicas de la Universidad Nacional de Trujillo. 1993.

18.- OLIVERA A, VALDERRAMA I. Investigación de la presencia de anticuerpos anti-Trypanosoma cruzi en pobladores del valle Jequetepeque, La Libertad-Perú. (Tesis Bachiller). Facultad de Ciencias Médicas de la Universidad Nacional de Trujillo. 1994.

19.- ESCALANTE H, ANGULO-PLASENCIA M. Frecuencia de pobladores del distrito de Chilete (Cajamarca) con serología positiva a Trypanosoma cruzi. Bol Peruano Parasit 1995; 11: 7-9.

20.- ARAGOT DE ROSELL R, RODRÍGUEZ A M, DE JESÚS R R et al. Tripomastigotes de sangre y cultivo celular de Trrypanosoma cruzi Y. II. Patología de la enfermedad de Chagas en ratones BALB/c. Parasitol al Día 2000; 24: 28-35.

21.- CARRASCO L. Alteraciones histopatológicas del sistema nervioso central de Mus musculus cepa BALB/c infectado con Trypanosoma cruzi del valle de Reque, Lambayeque-Perú. (Tesis Bachiller) Facultad de Ciencias Biológicas de La Universidad Nacional de Trujillo. 1993.

22.- ARIAS A. Patogenia de Mus musculus producido por Trypanosma cruzi aislado de Panstrongyfus chinai del norte peruano (Tesis Bachiller). Universidad Nacional de Trujillo. Trujillo-Perú. 1992.

23.- DOVOROK J, POSTON M. Estudio sobre la infección de ratones endogámicas con clones de Trypanosoma cruzi. Bol Of Sanit Panam 1987; 103: 93-105.

24.- ZÚÑIGAC, VARGASR, COURCELLES MT, VERGARA U. Infección experimental con Trypanosoma cruzi en macho y hembras de tres cepas de ratones. Parasitol al Día 1997; 21: 9-15.

25.- ROBBINS S, COTRN R. Patología estructural y funcional 3ra ed. Nueva Edit. Panom. México. 1987.

Agradecimientos: Al Dr. César Llerena del Laboratorio de Patología del Hospital Regional Docente de Trujillo (Perú) por la ayuda en la ejecución y lectura de los cortes histológicos.

\section{MEDICINE AND HEALTH IN THE TROPICS Marseille - France 11 - 15 September 2005}

XVIth International Congress for Tropical Medicine and Malaria

IVth European Congress on Tropical Medicine and International Health

VII ${ }^{\mathrm{e}}$ Congrès International de la Socièté de Pathologie Exotique

Centenaire de L'Institut de Médicine Tropicale du Service de Santè des Armèes 